\title{
Relevance of Systems Biology to Respiratory Medicine
}

Alvar Agustí, MD, PhD, FRCP, FERS $1,2,3$, Alejandra López-Giraldo, MD 1,2,3,4, Tamara Cruz, PhD $2,3,4$ and Rosa Faner, $\mathrm{PhD}^{2,3,4}$

${ }^{1}$ Pneumology Service Thorax Institute, Hospital Clinic, Barcelona, Spain; ${ }^{2}$ Institute for Biomedical Investigation August Pi i Sunyer IDIBAPS, Universitat de Barcelona, Barcelona, Spain; ${ }^{3}$ Centre for Biomedical Investigation Network of Respiratory Diseases CIBERES, Barcelona, Spain; ${ }^{4}$ Clinical Foundation for Biomedical Research, Hospital Clinic, Barcelona, Spain

\section{ABSTRACT}

Human biology is extremely complex because it is multi-level (i.e., it involves interactions between molecules, cells, tissues, and organs), redundant (i.e., there are multiple cross-talks between these elements), influenced by macro and/or micro environmental factors, and dynamic (i.e., it changes with time). Hence, the investigation of human health and disease requires a research strategy able to encompass this complexity. Systems biology and its human equivalent, network medicine, are novel research strategies that pursue, precisely, these goals. This review discusses the principal features of systems biology (Why bother? What is it? How do we do it?), and presents some examples of its application to network respiratory medicine using chronic obstructive pulmonary disease as a use-case. (BRN Rev. 2016;2:1-13)

Corresponding author: Alvar Agustí, alvar.agusti@clinic.ub.es

Key words: Complexity. Disease. Health. Modelling. Networks. 


\section{RATIONALE: WHY BOTHER?}

Human biology is extremely complex because it is: (i) multi-level, that is, it involves interactions between nucleic acids, proteins, metabolites, cells, tissues, and organs; (ii) redundant, that is, there are multiple cross-talk systems and over-abundant control systems within and between levels; (iii) strongly influenced by macro and/or micro environmental factors, which, importantly, can occur at each level of complexity; and, (iv) dynamic, that is, it varies with time in response to changes in both general and/or local milieu (i.e., environmental) conditions ${ }^{1,2}$. It is not surprising, therefore, that the two main "end products" of human biology, that is human health and disease, are also complex processes ${ }^{3}$. Hence, the investigation of human health and disease requires a research strategy able to encompass this complexity. To do so, it should firstly aim at understanding the complexity of each biological level (molecules, cells, organs), then consider their dynamic changes over time in response to local perturbations and, finally, encapsulate them all in a conceptual "system" that explains as closely as possible its behaviour ${ }^{3}$. These dynamic changes are often considered in the short-term (i.e., in response to a given acute intervention that perturbs the system), although a long-term dynamic view may also be of great interest, such as changes induced by ageing ${ }^{4-7}$. Systems biology ${ }^{8}$ and its human equivalent, network medicine ${ }^{9}$, are novel research strategies that pursue, precisely, a better and comprehensive understanding of all these aspects. This review discusses the principal features of systems biology and presents some examples of its application to network respiratory medicine.

\section{SYSTEMS BIOLOGY: WHAT IS IT?}

\section{Complex systems}

In the context of system biology, a complex system is a "collection of linked individual elements with emerging properties that cannot be attributed to each element considered separately"3. In biology, the "level" of a "complex system" so defined can range from the molecular, subcellular, and cellular level to organ and organism physiology or even the totality of environmental exposures or exposome ${ }^{10}$, and include all or part of these different levels ${ }^{1,2}$. To date, however, complex systems have been mostly studied at the cellular level ${ }^{11}$. Within this "organizational space", they have the following properties: (i) a hierarchical but concurrent nature of cellular functions ${ }^{11}$; (ii) adaptive dynamics that incorporate the ability to self-organize in response to transitions between healthy and pathological conditions ${ }^{11}$; (iii) non-linear relationships between the different elements of the system ${ }^{12,13}$; and, (iv) emergent properties, that is, one or more properties that cannot be attributed to any element of the system considered separately ${ }^{3,14}$. For instance, a plane is a complex (mechanical, not biological) system, composed of many different elements (or nodes), such as wings, engines, wheels, fuel, pilots, so on. that are linked in a specific manner (there are no planes with one or three wings). As such, the plane has one emergent property: it flies! Yet, none of its components can fly on its own. In order to fly, it is necessary that all the essential elements of the system (in this case, the plane) are there, linked in the appropriate manner. Admittedly though, there may be redundant (e.g. several engines) and non-essential parts (e.g. seats) 
also. Human health and disease are also emerging properties of an extraordinarily complex system: the human body. In the airplane example, however, engineers have designed and made the drawing plan of how all the necessary pieces are assembled together so it can fly. By contrast, in system biology we are doing (somewhat) the reverse process. We are identifying some of the individual components and trying to fit them and construct the building plan of the human body, not only anatomically but also considering its multilevel, functional detail.

\section{Network analysis}

A useful way to conceptualize and present graphically a complex "system" is a network, where the different elements of the system are represented as nodes and their structural and/or functional relationship as edges (or links) $)^{15}$. The "nature" (i.e., type) of the "nodes" and "edges" of a given system depends on the research question(s) we want to address. Hence, in different "systems", "nodes" can be genes, proteins, cells, organs, people, environmental factors (or a combination of them), or any other element we want to explore in our particular project. Likewise, how these nodes are "connected", that is, the type of links (also called edges) between nodes, can also vary according to our research interest. Thus, edges can represent structural or functional interactions between them (if nodes are molecules), co-occurrence of diseases (e.g., comorbidities), sharing friends, neighbourhood or environmental perturbations (e.g., smoking), or any other relationship that we want to explore. Further, edges can have "directionality" (if pertinent to the research question), that is, they can

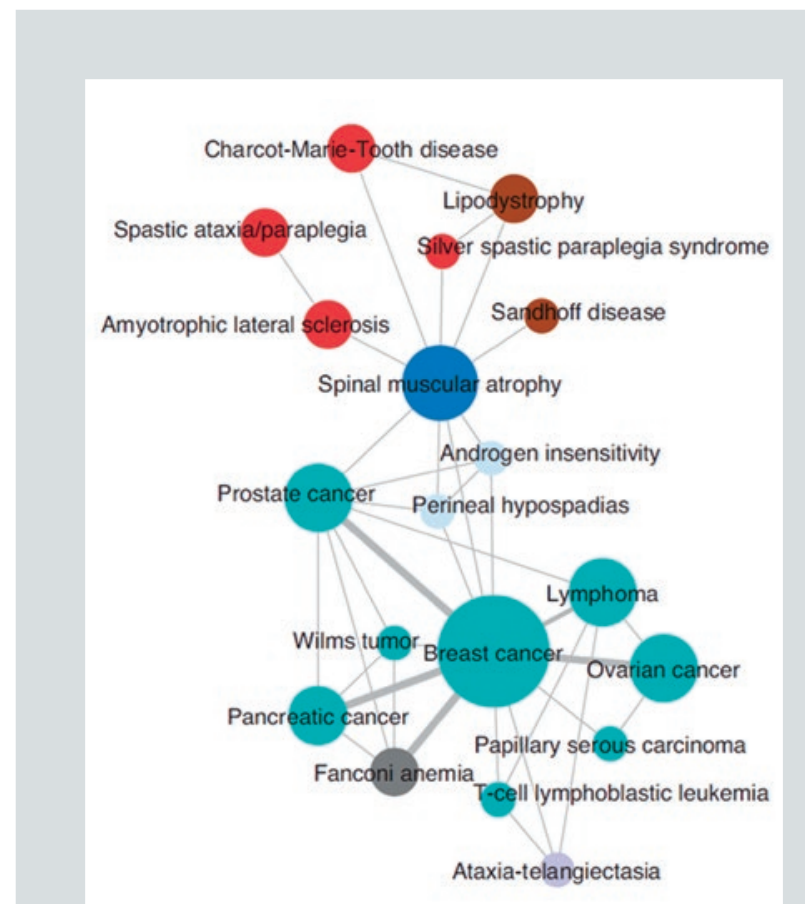

Figure 1. Example of a disease network (diseasome). Each node corresponds to one disease (see labels). The size of the node is proportional to the number of genes published to date that participate in that disease. The colour of each node corresponds to the disorder class to which the disease belongs. Two diseases are connected if they share one or more of these genes. The width of the link (edge) is proportional to the number of shared genes. For further explanations, see text (reproduced with permission $^{16}$.

indicate (arrow tip) the direction of how one node relates to the other (e.g., in a network of telephone users, who makes the phone call).

Figure 1 illustrates an important concept in systems biology: there is no such thing as a predefined network. Researches customize them depending on the specific questions they are pursuing. For instance, in figure 1 investigators were interested in understanding if different human diseases share associated genes (i.e., the gene network underlying them). Accordingly, they built a network (socalled, diseasome) where nodes correspond 


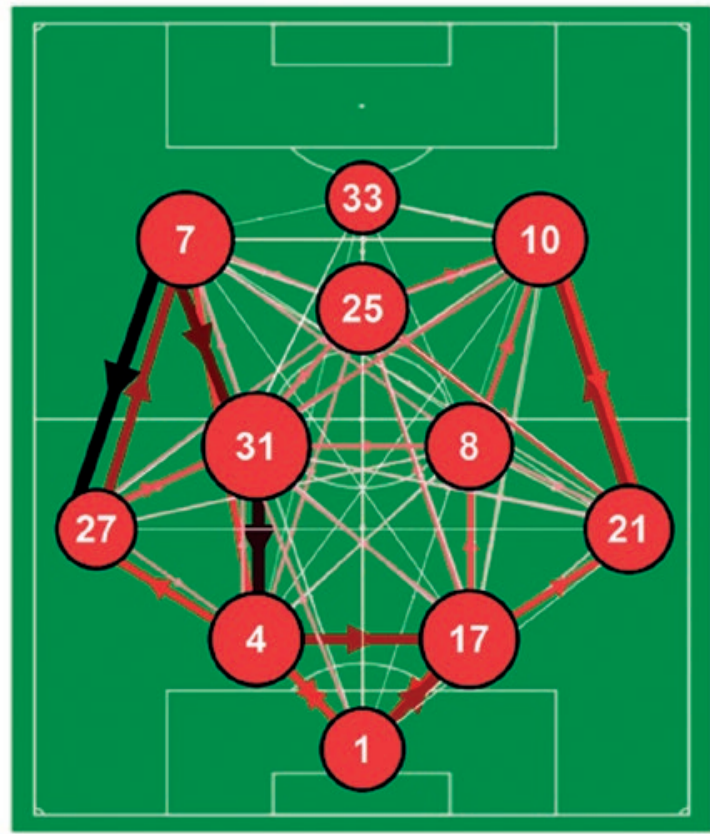

7 - Ribery, 33 - Gómez, 10 - Robben, 31 - Schweinsteiger, 25 - Müller, 8 - Martínez, 27 - Alaba, 4 - Dante, 17 - Boateng, 21 - Lahm, 1 - Neuer

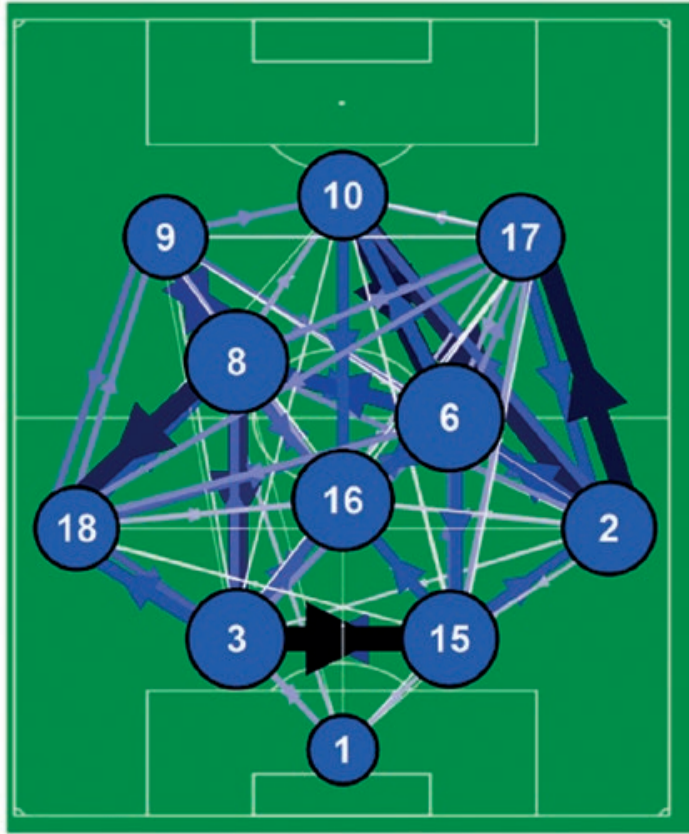

9 - Alexis, 10 - Messi, 17 - Pedro,

8 - Iniesta, 16 - Busquets, 6 - Xavi,

18 - Alba, 3 - Piqué, 15 -Bartra, 2 - Alves, 1 - Valdés

Figure 2. Passing network analysis of the Champions League football game between Bayern Munchen (red) and FC Barcelona (blue). Each node corresponds to one player (see names below the graph), links represent number of ball passes between each pair of them, the width of the link is proportional to the number of passes, and the arrow indicates the directionality of passes. For further explanations, see text (downloaded from http://2plus2equals11.wordpress.com/category/bayern-munich).

to diseases (their size is proportional to the number of genes that have been described in that particular disease, and their colour correspond to the disorder class to which the disease belongs) and edges (links) indicate that a particular gene is implicated in both diseases, whereas the width of a link is proportional to the number of genes that are implicated in both diseases ${ }^{16}$. By contrast, in figure 2 "investigators" (actually football coaches and specialized media) were interested in understanding the "dynamics" of a Champions league football game between Barcelona FC and Bayer Munich on April 23, 2013. Accordingly, they built their networks very differently. Here, each node corresponds to a given football player (see legend), the size of the node is proportional to the percentage of ball possession by the player, and links represent passes between them during the 90 minutes duration of the match (this is the dynamic component of a two-dimensional graph that can summarize temporal information), with the width of the link being proportional to the intensity (i.e., number of passes) of interaction between two given players, and the arrow indicating who made the pass and who received it (directionality). This network, therefore, represents a playing style (i.e., the dynamic structure of the team), and does not 


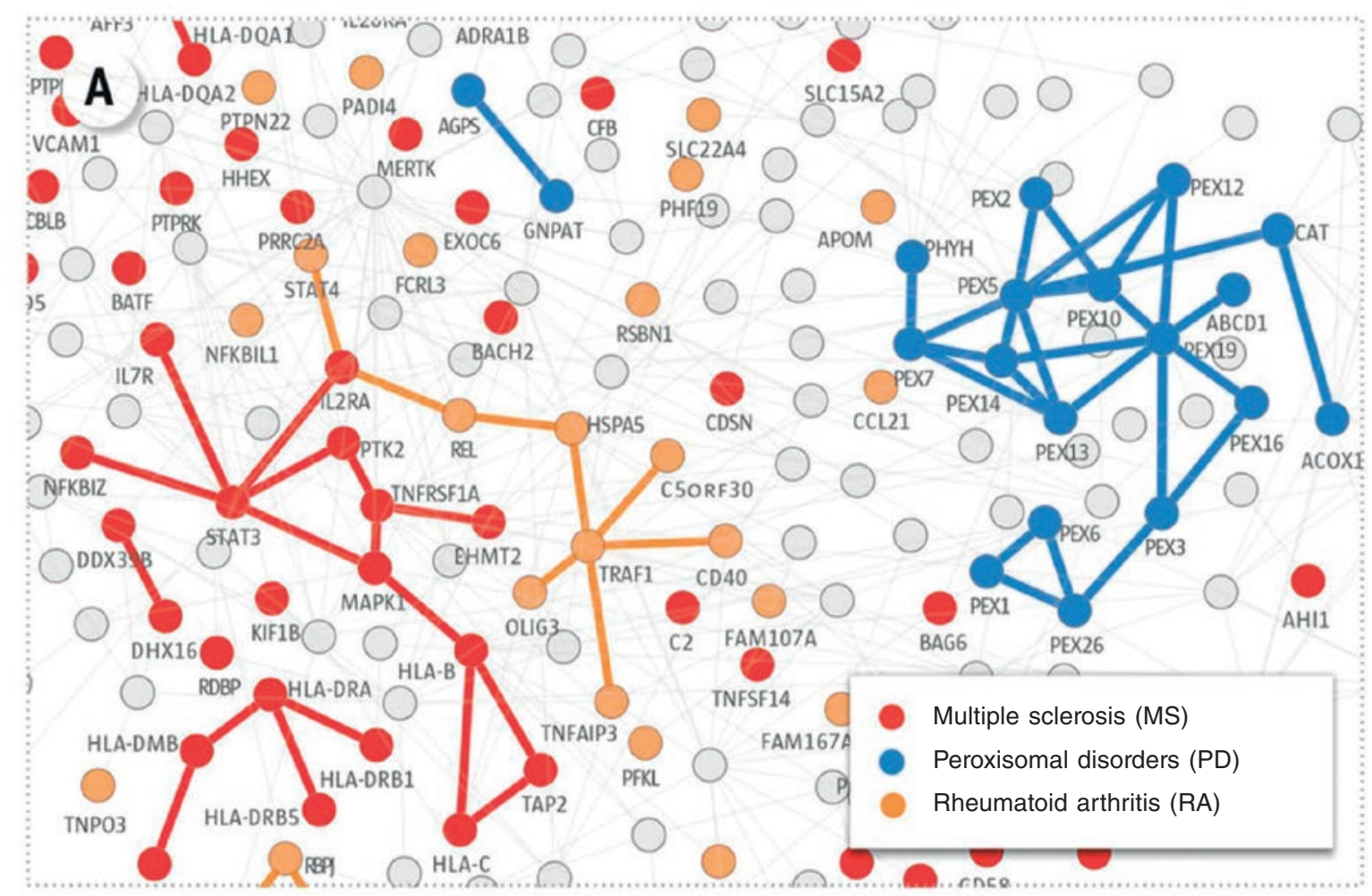

Figure 3. Disease modules identified in the full interactome between disease genes associated with the three diseases identified in the legend. For further explanations, see text. (reproduced with permission) ${ }^{18}$.

represent the match results (outcomes). These two completely different networks illustrate well the concept that network analysis provides a holistic and dynamic graphic representation of a complex system to facilitate the understanding of the relation between the different elements of the system. Actually, there is nothing new under the sun since for thousands of years we have known that "a picture is worth thousand words".

Given that complex systems are often understood and represented as networks, network analysis sought to: (i) unravel the relations between the different "elements" (nodes and edges) of the system; (ii) quantify and display graphically the topology (i.e., structure) of the network; and, (iii) investigate their main functional characteristics ${ }^{15}$. The latter are often studied by determining the presence of "functional modules"17-19. The disease module hypothesis proposes that the cellular components (genes, proteins, metabolites) associated with a given disease, segregate (i.e., are located) in the same "neighbourhood" (i.e., area) of the human interactome, the map (network) of biologically relevant molecular interactions (Fig. 3$)^{18}$. In fact, using novel mathematical modelling, it has been recently confirmed that the network-based location of each disease module determines its pathobiological relationship to other diseases ${ }^{18}$. Interestingly, diseases with overlapping network modules show significant co-occurrence (comorbidity) ${ }^{18}$. 


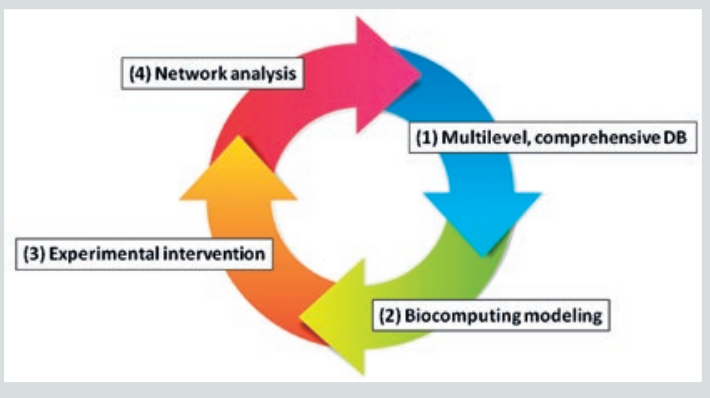

Figure 4. Iterative working phases of systems bio-medicine. DB: data base. For further explanations, see text.

\section{SYSTEMS BIOLOGY: HOW DO WE DO IT?}

Systems biology sought to: (i) integrate data within and between different levels of biological complexity; and (ii) develop mathematical "models" of the system that can explain its emergent properties and response to external perturbations $\mathrm{s}^{7,818,20,21}$. To achieve these goals, systems biology follows an iterative research strategy (Fig. 4) that generally involves the following steps:

- Generate a multi-level, comprehensive database from high-throughput genomics, transcriptomics, proteomics, and/or metabolomics platforms (albeit other types of data, such as the physiome or the clinicome) in one or more biological samples (e.g., blood and sputum) of interest in well-controlled model systems (cell culture, experimental animals) or phenotypically well-characterized individuals (including, if possible, clinical, functional, and imaging data). These data can be complemented with information existing in publically available databases through knowledge management platforms;
TABLE 1. Selected software tools for network analysis from two popular packages (Cytoscape (http://apps.cytoscape.org) and Bioconductor (http://www.bioconductor.org/ packages/ release/), although many others exist. Additional information for other software tools for network analysis in $\mathrm{R}$ can be found at CRAN (http://www.r-project.org)

\begin{tabular}{|c|c|c|}
\hline Name & Platform & Purpose \\
\hline $\begin{array}{l}\text { bayelvi- } \\
\text { raApp }\end{array}$ & Cytoscape & Bayesian network inference \\
\hline $\begin{array}{l}\text { jActive- } \\
\text { Modules }\end{array}$ & Cytoscape & Identification of network modules \\
\hline $\begin{array}{l}\text { CyTar- } \\
\text { getLinker }\end{array}$ & Cytoscape & $\begin{array}{l}\text { Link regulatory (transcription } \\
\text { factor) information }\end{array}$ \\
\hline DisGeNET & Cytoscape & Query disease-gene networks \\
\hline minet & Bioconductor & $\begin{array}{l}\text { Inference of networks using mutual } \\
\text { information }\end{array}$ \\
\hline birta & Bioconductor & Bayesian network inference \\
\hline BioNet & Bioconductor & Identification of network modules \\
\hline rTRM & Bioconductor & $\begin{array}{l}\text { Identification of transcriptional } \\
\text { regulatory modules }\end{array}$ \\
\hline Cyrface & Cytoscape & Interface between Cytoscape and R \\
\hline RCytoscape & Bioconductor & Interface between Cytoscape and R \\
\hline
\end{tabular}

Reproduced with permission ${ }^{15}$. References for the specific applications mentioned in the table can be found ${ }^{15}$.

- Apply biocomputing algorithms to this database to generate predictive mathematical models;

- Perturbate the system through some sort of experimental intervention (change in environmental conditions, molecular interference, genetic knock-out, pharmacological treatment, exercise training, diet intervention, other) and quantify and record multi-level comprehensive data again;

- Use network analysis to compare the response observed experimentally to the response predicted by the mathematical model. Table 1 lists a number of selected software tools for network analysis from 
two of the most popular packages (Cytoscape (http://apps.cytoscape.org) and Bioconductor (http:// www.bioconductor.org/ packages/release/), although many more are available ${ }^{15}$;

- Use the discrepancies between the predicted and observed responses to refine the model and start the cycle again until the predicted model fits the observed response reasonably well, in which case we will probably have a better understanding of the system and, therefore, on how to interfere with it for the purpose of improving its performance and/or repair its malfunction (i.e., disease).

\section{SYSTEMS BIOLOGY: WHAT DOES IT DELIVER?}

Systems biology has the potential to deliver a holistic, integrative, and dynamic view of complex biological conditions. Rather than focusing on a specific molecule (gene, protein, metabolite) at a given time, it presents a global perspective of all the potential molecules and pathways involved (and how they change in response to a perturbation of the system), which clearly gives a better representation of reality. In theory, this holistic, integrative, and dynamic view does not need to be restricted to the molecular biological level and could be used also at the cell level, organ level, or even combining different levels in the same analysis. Multi-level profiling of high-throughput molecular platforms in combination with clinical profiles (i.e., multi-level systems biology) is obviously much more informative but, at the same time, much more complex ${ }^{11}$, so most currently available publications of applications of systems biology to human diseases focus on the molecular level. The methodology, application, and proper validation of this multi-level approach is still, for the most part, in its infancy ${ }^{11}$. Further, it is absolutely necessary to validate such complex results rigorously. This very important area has only recently begun to be addressed ${ }^{22-24}$.

Systems approaches to biology and medicine are relatively new. Thus, this strategy is still mostly within the research domain. However, there are a few examples of successful practical applications both outside and within the respiratory field. Outside the respiratory field, the use of network analysis in neurodegenerative diseases, for instance, has already identified the autophagy pathway as a central pathogenic process ${ }^{25}$ and has identified potential novel therapeutic targets ${ }^{26}$. Within the respiratory field, there are some examples (Table 2) of systems biology approaches applied in the study of asthma, chronic obstructive pulmonary disease (COPD), cystic fibrosis, lung cancer, and pulmonary hypertension ${ }^{15}$. In the text that follows, we focus on COPD as a use case. Figure 5 presents a conceptual, multi-level network perspective of COPD, and the type of clinically relevant information that each of these levels will be able, eventually, to produce ${ }^{27}$. In this model, COPD is envisaged as a four-story building which, of course, is only a pictorial, simplified representation of this building and by no means pretends to be an accurate or comprehensive representation of reality. With this caveat in mind, it is worth noting that practicing physicians are used to working on the "blue" level (clinical network) of the building where they often encounter in their daily practice patients who suffer not only the index disease 
TABLE 2. Applications of network analysis to the study of respiratory diseases

\begin{tabular}{|c|c|c|}
\hline Disease & Method used & Primary finding \\
\hline Asthma & Transcriptome + Bayesian networks & TGF- $\beta 1$ is a key regulator of IL13-dependent asthma response \\
\hline Asthma & Transcriptome + PPI network & PPAR and INF pathways regulate response to glucocorticoids \\
\hline Asthma & SNPs + PPI network & $\begin{array}{l}\text { Identified non-synonymous SNPs in the coding sequences of the toll-like } \\
\text { receptor network }\end{array}$ \\
\hline Asthma & OMIM + transcriptome + PPI network & $\begin{array}{l}\text { Suggests that GNB2L1 plays a role as an important signalling mediator } \\
\text { in asthma }\end{array}$ \\
\hline Asthma and smoking & Transcriptome + IPA & TIMP1 and TSBH1 are related to oxidative stress \\
\hline COPD & COPD comorbidities (expert) + PPI & COPD multi-morbidities share genes and biological pathways \\
\hline COPD & Transcriptome + mutual information & COPD is associated with failure to regulate bioenergetics pathways \\
\hline COPD & Transcriptome + IPA & There is distinct gender specificity in response to acute smoking and COPD \\
\hline COPD & mRNA + methylation & There is a distinct epigenetic regulation in COPD that governs mRNA expression \\
\hline Lung cancer & PPI network & $\begin{array}{l}\text { Up-regulated genes tend to be highly connected, contrary to down-regulated } \\
\text { genes }\end{array}$ \\
\hline Multiple (diseasome) & OMIM & Shared genes suggest common origin for diseases \\
\hline $\begin{array}{l}\text { Pseudomonas aeruginosa } \\
\text { colonization in chronic } \\
\text { cystic fibrosis }\end{array}$ & Transcriptome + metabolome + KEGG & $\begin{array}{l}\text { Up-regulation of a pathogen's genes involved in adaptation during disease } \\
\text { progression represent potential therapeutic targets }\end{array}$ \\
\hline Pulmonary hypertension & $\begin{array}{l}\text { miRNA transcriptome }+ \text { target } \\
\text { prediction }\end{array}$ & Identified miRNA-21 as a regulator of disease pathways \\
\hline
\end{tabular}

IPA: ingenuity pathway analysis (QIAGEN), commercial software for network/pathway analysis; KEGG: Kyoto Encyclopaedia of Genes and Genomes; OMIM: Online Mendelian Inheritance in Man; PPI: protein-protein interactions; SNP: single-nucleotide polymorphism; TGF: transforming growth factor. The diseasome contains information related to several respiratory diseases, including asthma, COPD, and lung cancer ${ }^{15}$.

Reproduced with permission ${ }^{15}$. References for the specific applications mentioned in the table can be found in ${ }^{15}$.

(COPD in this case), but also several other diseases (comorbidities, which can be seen as the disease index by other colleagues (e.g., cardiologist, oncologist, etc.). Below this "blue" level, the COPD "building" has an "orange" level (biological network) where several biological process occur and interact (i.e., cross-talk) constantly, including innate and acquired immunity, oxidative stress, ageing, bioenergetics, repair processes, and others. In the basement of the COPD building there is the genetic level (network) where multiple interactions also occur between genes (DNA), RNA, and epigenetics (Fig. 5). In the roof of the building there is an environmental network (i.e., exposome, a term that describes the "totality of human environmental exposures, from conception onwards"10) that includes the interactions and mutual influences of temperature, humidity, pollution (including smoking), allergens, viruses, bacteria, and others (Fig. 5). Being the interactions of all these elements of crucial importance at each level (environment, clinical, biological, and genetic), we should not forget that they interact also in a supra-level network. For instance, changes in the environment (green level) can influence directly changes in the yellow level (genetic) through so-called "epigenetics"28. So this building is complex at each level, between levels and, importantly, changes with time, that is, it has a dynamic component (e.g., 


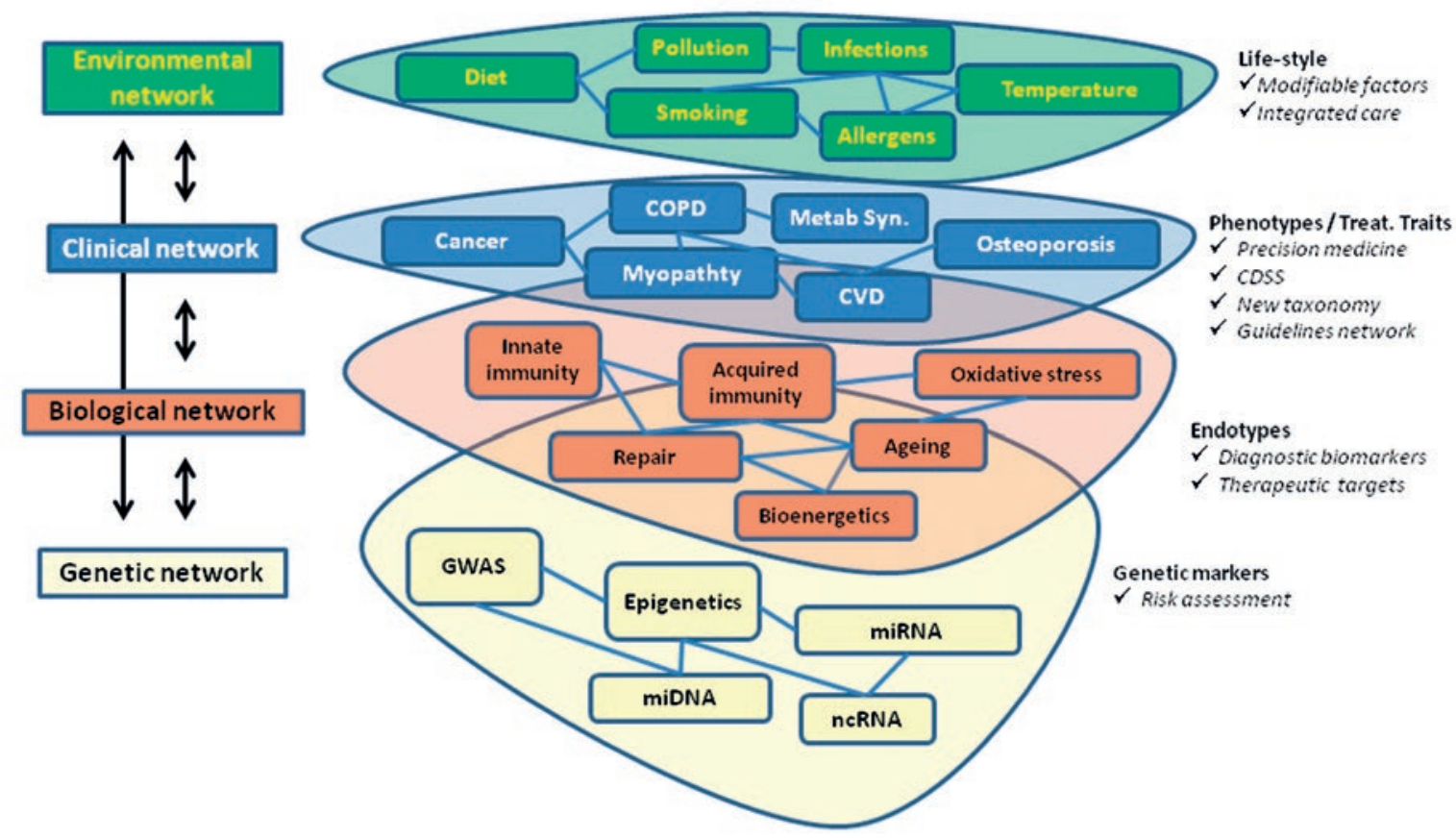

Figure 5. Schematic representation of the "COPD building" is formed by four different "floors"; each one of them is a complex network in itself. The "elevators" of the building (arrows on the left) connect (directly or indirectly) different levels of complexity. The right hand column lists a number of potential clinical benefits derived (eventually) from each "floor". For further explanations, see text. CVD: cardiovascular disease; CDSS: clinical decision support systems; GWAS: genome-wide association study (reproduced with permission $)^{27}$.

changes with an exacerbation of the disease and/or after treatment).

A better understanding of each of these four levels can potentially produce information of clinical relevance. The genetic level will be able to identify genetic markers that can help clinicians to better determine the future risk of an individual patient (risk of deterioration of the disease and/or development of complications and/or response to a given therapy) ${ }^{27}$. The biological level will eventually provide relevant information on "biological" markers that can help clinicians in their assessment and monitoring of a given patient. A deeper understanding of the relationships between different comorbidities in the clinical level 29,30 can facilitate better strategies for integrated care $^{31,32}$, the development of so-called "clinical decision support systems" (CDSS) ${ }^{2}$, such as the COPD control panel ${ }^{13}$, which provides a way to visualize the complexity of COPD, and the combined assessment of the severity, impact, and activity to best inform the physician on the most appropriate management strategies for an individual patient based on the "treatable traits" present in this particular patient at this specific time point ${ }^{13}$. Importantly, the control panel can be customized to the needs of the patient and the resources available locally (e.g., rural vs. urban healthcare centres; primary vs. specialized care). 
This clinical network may also stimulate the eventual development of a network of guidelines that facilitate the assessment and treatment of COPD patients. Finally, interventions at the level of the "exposome" will promote healthier life-styles and preventive measures.

The above discussion is a conceptual (i.e., theoretical) one. Yet we and others have already applied network analysis to investigate several aspects of COPD at all four levels (environmental, clinical, biological, and genetic) of complexity (Fig. 5). For instance, we explored the molecular and environmental relationships between the comorbid diseases that occur frequently in COPD patients ${ }^{30}$. To this end, we followed a two-step research strategy. First, we used data mining (that is, exploitation of data published in the literature) and developed a novel, unbiased, integrative network analysis approach for the integrated analysis of the diseasome (i.e., relationships between comorbidities), interactome (i.e., molecular relationships), the involved biological pathways (i.e., functional biochemical implications) and tobacco smoke exposome of 16 prevalent COPD comorbidities $^{30}$. Main results showed that: (i) all 16 COPD comorbidities were related at the molecular level because they shared genes, proteins, and biological pathways such as inflammation, endothelial dysfunction, or apoptosis; (ii) there were previously overlooked biological pathways that may also contribute to COPD comorbidities, such as hemostasis, depression, and cell cycle abnormalities; and, (iii) finally, that chemicals contained in the tobacco smoke target about $70 \%$ of the identified proteins participating in COPD comorbidities ${ }^{30}$. All in all, this data mining analysis allowed the identification of plausible molecular links between
COPD and comorbid diseases, and showed that many of them are targets of the tobacco exposome ${ }^{30}$. Then, we extended this data mining analysis to real world data by using information gathered from two large COPD clinical audits ${ }^{33-37}$ to study the clinical and molecular relationships between comorbidities in 5,447 patients hospitalized because of an exacerbation of $\mathrm{COPD}^{38}$. In these patients we constructed and compared their clinical diseasome (CD) and the molecular diseasome (MD). The former (CD) is a network representation of the relationships between comorbid diseases, where diseases are linked if they co-occur more than expected at random, whereas in the latter (MD), diseases are linked if they share associated genes or interaction between proteins ${ }^{38}$. We observed that about half of the disease pairs identified in the $\mathrm{CD}$ had a biological counterpart in the MD, particularly related to inflammation and vascular tone regulation ${ }^{38}$, further supporting the existence of shared molecular mechanisms among comorbidities in $\mathrm{COPD}^{38,39}$. Interestingly, the $\mathrm{CD}$ of these patients appears independent of age, cumulative smoking exposure or severity of airflow limitation ${ }^{38}$.

Menche et al. ${ }^{17}$ developed a novel unbiased method (diVIsive Shuffling Approach or VIStA) that identifies subgroups (i.e., phenotypes) of COPD patients by maximizing the difference in their gene expression patterns (genetic, biological, and clinical levels in figure 5). Using this methodology in 140 COPD patients included in the ECLIPSE cohort ${ }^{40-42}$, these investigators identified four distinct, biologically and clinically meaningful combinations of clinical, functional, imaging, and biological characteristics that are associated with large gene expression differences (Fig. 6) ${ }^{17}$. 


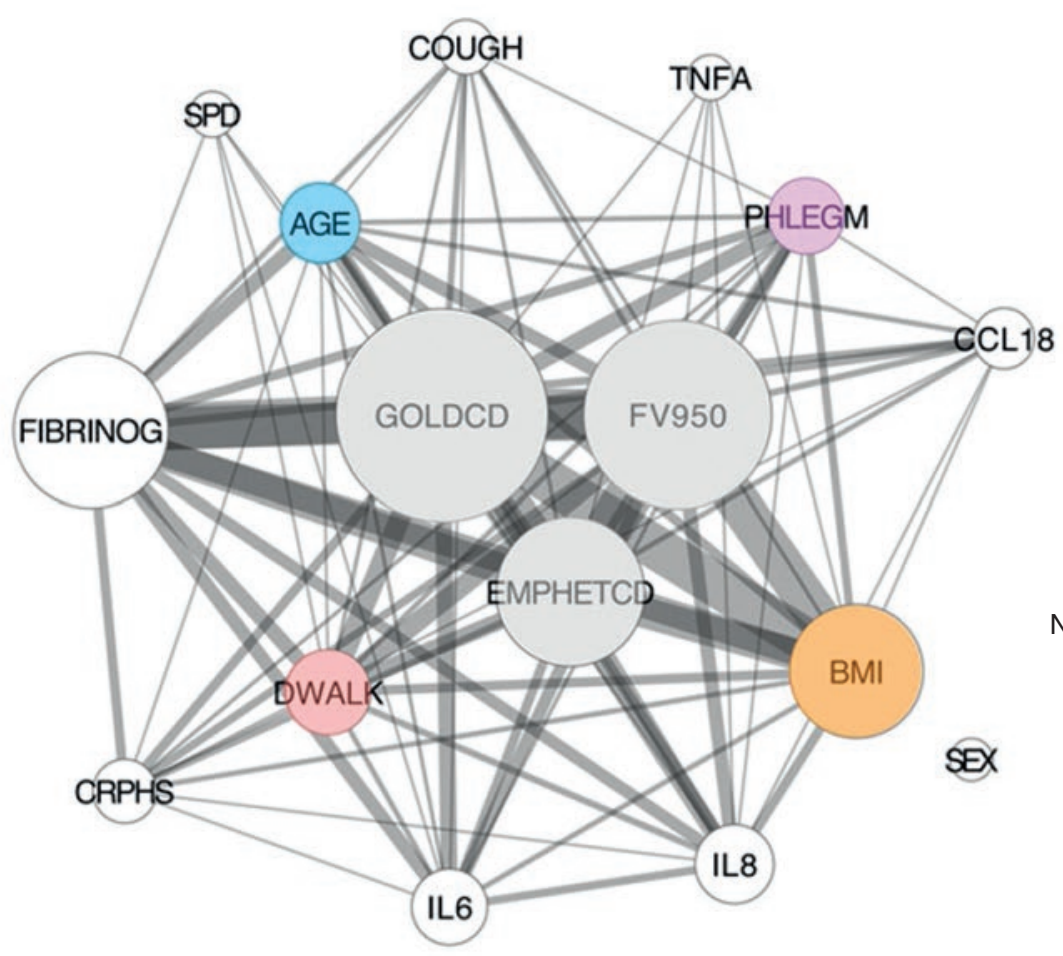

Number of times single clinical characteristic was found significant characteristics were found significant together

FIgURE 6. Network representation of the related clinical, functional, imaging or biological characteristics identified by VIStA. As indicated by legends, node size is proportional to the number of times a given characteristic was found significant in VIStA; the width of a link indicates how often two given characteristics were found significant together. The core group of the network contains severity of airflow limitation (GOLDCD) and the two measures of emphysema severity (EMPHETCD and FV950). Other characteristics included in the network were: age, gender (SEX), chronic bronchitis (PHLEGM), cough, body mass index, six-minute walking distance (DWALK) and several biomarkers (TNF- $\alpha$, CCL18, IL8, IL6, C-reactive protein (CRPHS), fibrinogen (FIBRINOG) and surfactant protein D. For more explanations, see text. BMI: body mass index; SPD: surfactant protein D; TNF- $\alpha$ : tumour necrosis factor alpha; IL: interleukin (reproduced with permission)17.

Given that tobacco smoking is the main risk factor of COPD, that not all smokers develop the disease, and that an abnormal pulmonary and systemic inflammatory response to smoking is thought to play a major pathogenic role ${ }^{43-45}$, Faner et al. reasoned that smokers with COPD should develop a different inflammatory response than that of smokers without COPD after acute smoking ${ }^{46}$. To explore this hypothesis (biological and genetic levels in figure 5), they used network analysis to characterized the systemic leukocyte transcriptomic response to smoking in smokers with and without $\mathrm{COPD}^{46}$. Results identified two main observations: (i) there were remarkable differences in the leukocyte transcriptomic response to acute smoking in males and females; and, (ii) in both genders, we identified genes, ontologies and interaction networks that were differentially expressed in response to smoking exclusively in COPD patients (COPD-related signature) or smokers with normal spirometry (smoking-related signature) ${ }^{46}$.

Very recently Yoo et al. developed a systematic and integrative approach of genome-wide 
DNA methylation, gene expression, and phenotype data (genetic, biological and clinical levels in figure 5) in lung tissue from COPD and controls to identify key molecular regulators of $\mathrm{COPD}^{47}$. They identify 126 such regulators among which EPAS1 was the only one whose downstream genes significantly overlapped with multiple genes sets associated with COPD disease severity ${ }^{47}$. This study illustrates very well the power of systems biology approaches to understand the complexity of COPD and other respiratory (or human) diseases. Of course, from this perspective, systems biology should be seen as a hypothesis generating exercise, since findings and observations will have to be validated later using targeted interventions in appropriate experimental and/or clinical settings.

Finally, in this setting, the analysis of the microbiome represents a new era of exciting discoveries since it can include in the "system" genes "on us" but not "from us"48. This shows the grey area between self and nonself in life in general, and in health and disease in particular. To address this complexity, systems biology approaches will be needed. In fact, they have already begun to be applied to several respiratory diseases too ${ }^{49-55}$.

\section{CONCLUSIONS}

Systems biology and its medical counterpart network medicine are here to stay. They are research strategies aimed at investigating the pathobiological basis of health and disease from a holistic, integrative view. Given the complexity of human biology discussed above, such strategy is absolutely needed if we are to really make progress towards a more efficient and safe, personalized and precise medicine in the years to come con-56-65 $^{2,5 o}$ as sharply proposed by one of the anonymous reviewers of this paper, system biology is actually attempting to understand the master drawing that the creator had in mind. To this end, the young generation of researches will have to be a combination of Michelangelo, Copernicus and ... Gaudi!

\section{REFERENCES}

1. Kohl P, Crampin EJ, Quinn TA, Noble D. Systems biology: an approach. Clin Pharmacol Ther. 2010;88:25-33.

2. Agusti A, Anto JM, Auffray $\mathrm{C}$ et al. Personalized respiratory medicine: Exploring the horizon, addressing the issues. Am J Respir Crit Care Med. 2015;191:391-401.

3. Agusti A, Sobradillo P, Celli B. Addressing the complexity of chronic obstructive pulmonary disease: From phenotypes and biomarkers to scale-free networks, systems biology, and P4 medicine. Am J Respir Crit Care Med. 2011;183:1129-37.

4. Bolt K, Bergman A. Systems biology of aging. Adv Exp Med Biol. 2015;847:163-78.

5. Chauhan A, Liebal UW, Vera J et al. Systems biology approaches in aging research. Interdiscip Top Gerontol. 2015;40:155-76.

6. Hou L, Huang J, Green CD et al. Systems biology in aging: linking the old and the young. Curr Genomics. 2012;13:558-65.

7. Faner R, Cruz T, López-Giraldo A, Agusti A. Network medicine, multimorbidity and the lung in the elderly. Eur Respir J. 2014;44:775-88.

8. Kitano H, editor. Foundations of systems biology. Cambridge: The MIT Press; 2001.

9. Barabasi AL, Gulbahce N, Loscalzo J. Network medicine: a network-based approach to human disease. Nat Rev Genet. 2011;12:56-68.

10. Vrijheid M. The exposome: a new paradigm to study the impact of environment on health. Thorax. 2014;69:876-8.

11. Periyasamy S, Gray A, Kille P. The bottom-up approach to defining life: deciphering the functional organization of biological cells via multi-objective representation of biological complexity from molecules to cells. Front Physiol. 2013;4:369.

12. Seely AJ, Macklem P. Fractal variability: an emergent property of complex dissipative systems. Chaos. 2012;22:013108.

13. Agusti A, MacNee W. The COPD control panel: towards personalised medicine in COPD. Thorax. 2013;68:687-90.

14. Macklem PT. Emergent phenomena and the secrets of life. J Appl Physiol. 2008;104:1844-6.

15. Diez D, Agusti A, Wheelock CE. Network analysis in the investigation of chronic respiratory diseases: from basics to application. Am J Respir Crit Care Med. 2014;190:981-8.

16. Goh KI, Cusick ME, Valle D, Childs B, Vidal M, Barabasi AL. The human disease network. Proc Natl Acad Sci U S A. 2007;104:8685-90.

17. Menche J, Sharma A, Cho M et al. A diVIsive Shuffling Approach (VIStA) for gene expression analysis to identify subtypes in chronic obstructive pulmonary disease. BMC Syst Biol. 2014;8(Suppl 2):S8.

18. Menche J, Sharma A, Kitsak M et al. Disease networks. Uncovering disease-disease relationships through the incomplete interactome. Science. 2015;347:1257601.

19. Sharma A, Menche J, Huang CC et al. A disease module in the interactome explains disease heterogeneity, drug response and captures novel pathways and genes in asthma. Hum Mol Genet. 2015;24:3005-20. 
20. Wolkenhauer O, Auffray C, Jaster R, Steinhoff G, Dammann O. The road from systems biology to systems medicine. Pediatr Res. 2013;73:502-7.

21. Silverman EK, Loscalzo J. Network medicine approaches to the genetics of complex diseases. Discov Med. 2012;14:143-52.

22. McShane LM, Cavenagh MM, Lively TG et al. Criteria for the use of omicsbased predictors in clinical trials: explanation and elaboration. BMC Med. 2013;11:220-42.

23. McShane LM, Cavenagh MM, Lively TG et al. Criteria for the use of omicsbased predictors in clinical trials. Nature. 2013;502:317-20.

24. Collins GS, Reitsma JB, Altman DG, Moons KG. Transparent Reporting of a multivariable prediction model for Individual Prognosis Or Diagnosis (TRIPOD). Ann Intern Med. 2015;162:735-6.

25. Caberlotto L, Nguyen TP. A systems biology investigation of neurodegenerative dementia reveals a pivotal role of autophagy. BMC Syst Biol. 2014;8:65.

26. Nguyen TP, Priami C, Caberlotto L. Novel drug target identification for the treatment of dementia using multi-relational association mining. Sci Rep. 2015;5:11104.

27. Agusti A, Vestbo J. Current controversies and future perspectives in chronic obstructive pulmonary disease. Am J Respir Crit Care Med. 2011; 184:507-13.

28. Adcock Im, Ford P, Barnes PJ, Ito K. Epigenetics and airways disease. Respir Res. 2006;7:21.

29. Divo M, Cote C, de Torres JP et al. Comorbidities and risk of mortality in patients with chronic obstructive pulmonary disease. Am J Respir Crit Care Med. 2012;186:155-61.

30. Grosdidier S, Ferrer A, Faner R et al. Network medicine analysis of COPD multimorbidities. Respir Res. 2014;15:111.

31. Bousquet J, Anto J, Sterk P et al. Systems medicine and integrated care to combat chronic noncommunicable diseases. Genome Med. 2011;3:43-55.

32. Bousquet J, Addis A, Adcock I et al. Integrated care pathways for airway diseases (AIRWAYS-ICPs). Eur Respir J. 2014;44:304-23.

33. Pozo-Rodriguez F, Alvarez CJ, Castro-Acosta A et al. [Clinical audit of patients admitted to hospital in Spain due to exacerbation of COPD (AUDIPOC study): method and organisation]. Arch Bronconeumol. 2010; 46:349-57.

34. Pozo-Rodriguez F, Lopez-Campos JL, Alvarez-Martinez CJ et al. Clinical Audit of COPD Patients Requiring Hospital Admissions in Spain: AUDIPOC Study. PLoS One. 2012;7:e42156.

35. Lopez-Campos JL, Hartl S, Pozo-Rodriguez F, Roberts CM. European COPD Audit: design, organisation of work and methodology. Eur Respir J. 2013;41:270-6.

36. Lopez-Campos JL, Hartl S, Pozo-Rodriguez F, Roberts CM. Variability of hospital resources for acute care of COPD patients: the European COPD Audit. Eur Respir J. 2014;43:754-62.

37. Pozo-Rodrguez F, Castro-Acosta A, Alvarez CJ et al. Determinants of between-hospital variations in outcomes for patients admitted with COPD exacerbations: findings from a nationwide clinical audit (AUDIPOC) in Spain. Int J Clin Pract. 2015;69:938-47.

38. Faner R, Gutierrez-Sacristan A, Castro-Acosta A, Grosdidier S, Gan W, Sanchez-Mayor M et al. Molecular and clinical diseasome of comorbidities in exacerbated COPD patients. Eur Respir J. 2015;46:1001-10.

39. Faner R, Agusti A. Network analysis: a way forward to understand COPD multimorbidity. Eur Respir J. 2015;46:591-2.

40. Vestbo J, Anderson W, Coxson HO et al. Evaluation of COPD Longitudinally to Identify Predictive Surrogate End-points (ECLIPSE). Eur Respir J. 2008;31:869-73.

41. Singh D, Edwards L, Tal-Singer R, Rennard S. Sputum neutrophils as a biomarker in COPD: findings from the ECLIPSE study. Respir Res. 2010;11:77.

42. Singh D, Fox SM, Tal-Singer R, Bates S, Riley JH, Celli B. Altered gene expression in blood and sputum in COPD frequent exacerbators in the ECLIPSE cohort. PLoS One. 2014;9:e107381.
43. Fletcher $\mathrm{C}$, Peto R. The natural history of chronic airflow obstruction. Br Med J. 1977;1:1645-8

44. Vestbo J, Hurd SS, Agusti A et al. Global strategy for the diagnosis, management and prevention of chronic obstructive pulmonary disease, GOLD Executive Summary. Am J Respir Crit Care Med. 2013;187:347-65.

45. Lange P, Celli B, Agusti A et al. Lung-function trajectories leading to chronic obstructive pulmonary disease. N Engl J Med. 2015;373:111-22.

46. Faner R, Gonzalez N, Cruz T, Kalko SG, Agusti A. Systemic inflammatory response to smoking in chronic obstructive pulmonary disease: evidence of a gender effect. PLoS One. 2014;9:e97491.

47. Yoo S, Takikawa S, Geraghty P et al. Integrative analysis of DNA methyl ation and gene expression data identifies EPAS1 as a key regulator of COPD. PLoS Genet. 2015;11:e1004898.

48. Kiley JP, Caler EV. The lung microbiome. A new frontier in pulmonary medicine. Ann Am Thorac Soc. 2014;11(Suppl 1):S66-70.

49. Sze MA, Dimitriu PA, Suzuki M et al. Host response to the lung microbiome in chronic obstructive pulmonary disease. Am J Respir Crit Care Med. 2015;192:438-45.

50. Sethi S. Chronic obstructive pulmonary disease and infection. Disruption of the microbiome? Ann Am Thorac Soc. 2014;11(Suppl 1):S43-7.

51. Segal LN, Rom WN, Weiden MD. Lung microbiome for clinicians. New discoveries about bugs in healthy and diseased lungs. Ann Am Thorac Soc 2014;11:108-16

52. Han MK, Zhou Y, Dickson R et al. Lung microbiome predicts disease progression in idiopathic pulmonary fibrosis. Ann Am Thorac Soc. 2014;11(Suppl 1):S79-80

53. Sze M, Dimitriu PA, Suzuki M et al. Host response to the lung microbiome in lung tissue undergoing emphysematous destruction. Ann Am Thorac Soc. 2014;11(Suppl 1):S77.

54. Demoruelle MK, Norris JM, Holers VM, Harris JK, Deane KD. The lung microbiome differs in asymptomatic subjects at elevated risk of future rheumatoid arthritis compared with healthy control subjects. Ann Am Thorac Soc. 2014;11(Suppl 1):S74.

55. Segal LN, Alekseyenko A, Clemente JC et al. Enrichment of lung microbiome with supraglotic microbes is associated with increased pulmonary inflammation. Ann Am Thorac Soc. 2014;11(Suppl 1):S71.

56. Collins FS, Varmus H. A new initiative on precision medicine. N Engl Med. 2015;372:793-5.

57. Ritchie MD, de Andrade M, Kuivaniemi H. The foundation of precision medicine: integration of electronic health records with genomics through basic, clinical, and translational research. Front Genet. 2015;6:104.

58. Jameson JL, Longo DL. Precision medicine - personalized, problematic, and promising. N Engl J Med. 2015;372:2229-34

59. Mirnezami R, Nicholson J, Darzi A. Preparing for precision pedicine. N Eng J Med. 2012;366:489-90.

60. Agusti A. The path to personalized medicine in COPD. Thorax. 2014;69:857-64

61. Auffray C, Hood L. Editorial: Systems biology and personalized medicine - the future is now. Biotechnol J. 2012;7:938-9.

62. Sznajder JI, Ciechanover A. Personalized Medicine. The road ahead. Am Respir Crit Care Med. 2012;186:945-7.

63. Holgate ST, Palotie A, Prainsack B et al. Personalized medicine for the European Citizen. 2012. European Science Foundation Forward Look Available at: http://www.esf.org/fileadmin/Public_documents/Publications/Personalised_Medicine.pdf

64. Loscalzo J. Systems biology and personalized medicine: a network approach to human disease. Proc Am Thorac Soc. 2011;8:196-8.

65. Galas DJ, Hood L. Systems biology and emerging technologies will catalyze the transition from reactive medicine to predictive, personalized, preventive and participatory (P4) medicine. IBC. 2009;1:1-5. 